\title{
Postoperative Spinal Subdural Lesions Following Lumbar Spine Surgery: Prevalence and Risk Factors
}

\author{
Yukitaka Nagamoto ${ }^{1}$, Shota Takenaka ${ }^{2}$, Hiroyuki Aono ${ }^{1}$ \\ ${ }^{I}$ Department of Orthopaedic Surgery, National Hospital Organization Osaka Medical Center, Osaka, Japan \\ ${ }^{2}$ Department of Orthopaedics, Osaka University Graduate School of Medicine, Osaka, Japan
}

\section{Study Design: Retrospective case-control study}

Purpose: To clarify the prevalence and risk factors for spinal subdural lesions (SSDLs) following lumbar spine surgery.

Overview of Literature: Because SSDLs, including arachnoid cyst and subdural hematoma, that develop following spinal surgery are seldom symptomatic and require reoperation, there are few reports on these pathologies. No study has addressed the prevalence and risk factors for SSDLs following lumbar spine surgery.

Methods: We conducted a retrospective analysis of the magnetic resonance (MR) images and medical records of 410 patients who underwent lumbar decompression surgery with or without instrumented fusion for degenerative disorders. SSDLs were classified into three grades: grade 0 , no obvious lesion; grade 1, cystic lesion; and grade 2, lesions other than a cyst. Grading was based on the examination of preoperative and postoperative MR images. The prevalence of SSDLs per grade was calculated and risk factors were evaluated using multivariate logistic regression analysis.

Results: Postoperative SSDLs were identified in 123 patients (30.0\%), with $50(12.2 \%)$ and $73(17.8 \%)$ patients being classified with grade 1 and 2 SSDLs, respectively. Among these, one patient was symptomatic, requiring hematoma evacuation because of the development of incomplete paraplegia. Bilateral partial laminectomy was a significantly independent risk factor for SSDLs (odds ratio, 1.52; $95 \%$ confidence interval, $1.20-1.92 ; p<0.001)$. In contrast, a unilateral partial laminectomy was a protective factor (odds ratio, $0.11 ; 95 \%$ confidence interval, $0.03-0.46 ; p=0.002$ ).

Conclusions: The prevalence rate of grade 1 SSDLs was $30 \%$, with no associated clinical symptoms observed in all but one patient. Bilateral partial laminectomy increases the risk for SSDLs, whereas unilateral partial laminectomy is a protective factor.

Keywords: Spinal subdural hematoma; Arachnoid cysts; Surgery; Complications; Risk factor

\section{Introduction}

Spinal epidural hematomas are the most common cause of symptomatic neural compression during the early postoperative period following spinal surgery $[1,2]$. In contrast, spinal subdural hematoma (SSDH) is an extremely rare complication of spinal surgery, with only five cases having been reported in the literature [3-9]. Incidental durotomy and traumatic blunt dissection are primary causes of SSDH $[5,10,11]$. However, a comprehensive analysis of risk factors for SSDH has not been performed.

In our clinical practice, we experienced a case of SSDH following lumbar decompression with posterior instrumented fusion, which required hematoma evacuation

Received Dec 9, 2016; Revised Mar 2, 2017; Accepted Mar 7, 2017

Corresponding author: Yukitaka Nagamoto

Department of Orthopaedic Surgery, National Hospital Organization Osaka Medical Center, 2-1-14 Hoenzaka, Chuou-ku, Osaka 5400006, Japan

Tel: +81-6-6942-1331, Fax: +81-6-6943-6467, E-mail: 7gam0to@gmail.com 
because of the development of incomplete paraplegia. Following this case, we conducted a retrospective review of preoperative and postoperative magnetic resonance (MR) images of patients who had undergone lumbar spine surgery at Department of Orthopaedic Surgery, National Hospital Organization Osaka Medical Center, Osaka, Japan. Unexpectedly, we identified a non-negligible number of patients in whom a lesion within the dura mater had developed postoperatively, inducing a shift of the cauda equina. We defined these lesions as spinal subdural lesions (SSDLs). Based on our analysis of preoperative and postoperative MR images, this study provides an investigative overview of the prevalence and risk factors for SSDLs after lumbar spine surgery.

\section{Materials and Methods}

We conducted a retrospective review of the preoperative and postoperative MR images of 427 consecutive patients who underwent lumbar decompression surgery with or without instrumented fusion for degenerative disorders at our institution (2012 September-2015 October). Patients with vertebral fractures, spinal metastasis, and spinal infections were excluded from our analysis. Approval was obtained from our Institutional Review Board (IRB no., 15192).

At our institution, patients undergo preoperative MR imaging (MRI), with reimaging performed at approximately 7 days postoperatively to confirm adequate surgical decompression. The standard lumbar MRI protocol comprises sagittal T1- and T2-weighted sequences and an axial T2-weighted sequence. Among the 427 patients enrolled in the study, preoperative and postoperative MR images were not available for 17 patients. Therefore, our analysis was based on data of 410 patients (204 males and 206 females) with a mean age of $70.4 \pm 11.2$ years (range, 16-91 years) and a mean follow-up of $14.2 \pm 9.9$ months (range, 0-37 months). Postoperative MRI was performed at $6.8 \pm 1.3$ days (range, $2-14$ days) postoperatively. Among our study group, 158, 158, 43,21,13, and 17 patients underwent surgery for degenerative spondylolisthesis, spinal canal stenosis, lumbar disk herniation, spondylolytic spondylolisthesis, adjacent segment disease, and other lumbar spinal diseases, respectively. The following demographic and clinical factors were extracted from medical records for analysis: age, sex, height, weight, anticoagulant therapy, type of surgical procedure, previous surgery at the index level, incidental durotomy, length of surgery, estimated volume of blood loss, and dosage of diclofenac suppository used over the first 7 postoperative days. With respect to the surgical procedures, patients underwent two-level posterior lumbar interbody fusion (PLIF) with or without additional partial laminectomy $(n=29)$, onelevel PLIF with or without additional partial laminectomy $(n=208)$, bilateral partial laminectomy (BPL; $n=133)$, unilateral partial laminectomy (UPL; $n=7$ ), and UPL with discectomy (UPLWD; $n=33$ ). PLIF and partial laminectomy were performed according to standard procedures, as previously described $[12,13]$. Endoscopic partial laminectomy was performed via an unilateral approach [14].

\section{MRI-based grading of SSDLs}

We developed an MRI-based grading system for the diagnosis of SSDLs (Fig. 1). This system comprised three grades that were determined based on operative changes within the dura mater observed on preoperative and postoperative sagittal and axial T2- and sagittal T1-weighted images (Figs. 2-4). The three grades are defined as follows: grade 0 , no evidence of a space-occupying lesions (SOLs) within the dura mater; grade 1, presence of SOL with an intensity equivalent to that of cerebrospinal fluid (CSF; T1 hypo/T2 hyper); and grade 2, presence of SOL with an intensity different to that of CSF. Based on the intensity

MRI grading system for the diagnosis of SSDL

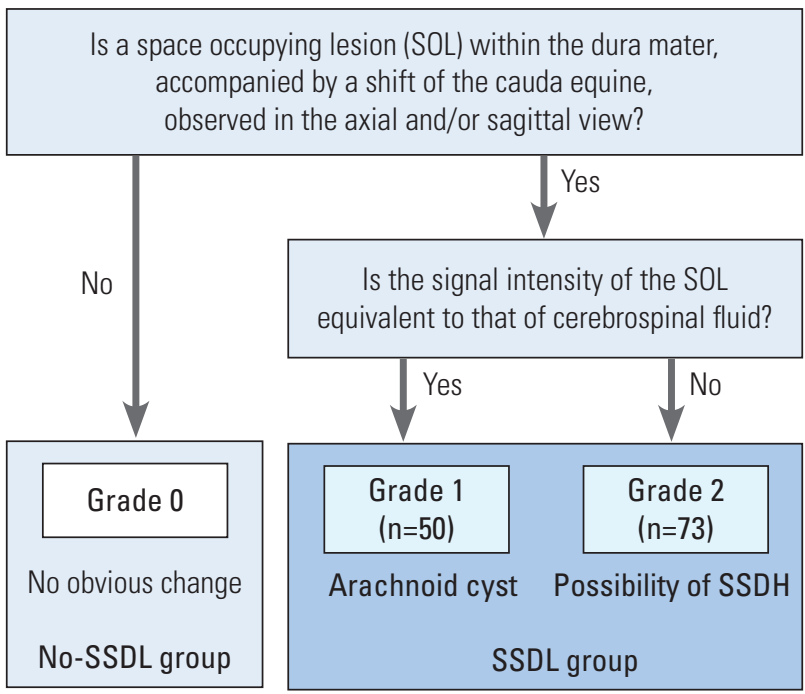

Fig. 1. Magnetic resonance imaging (MRI)-based grading system for the diagnosis of spinal subdural lesions (SSDLs) based on preoperative to postoperative changes within the dura mater. The three SSDL grades are depicted. 

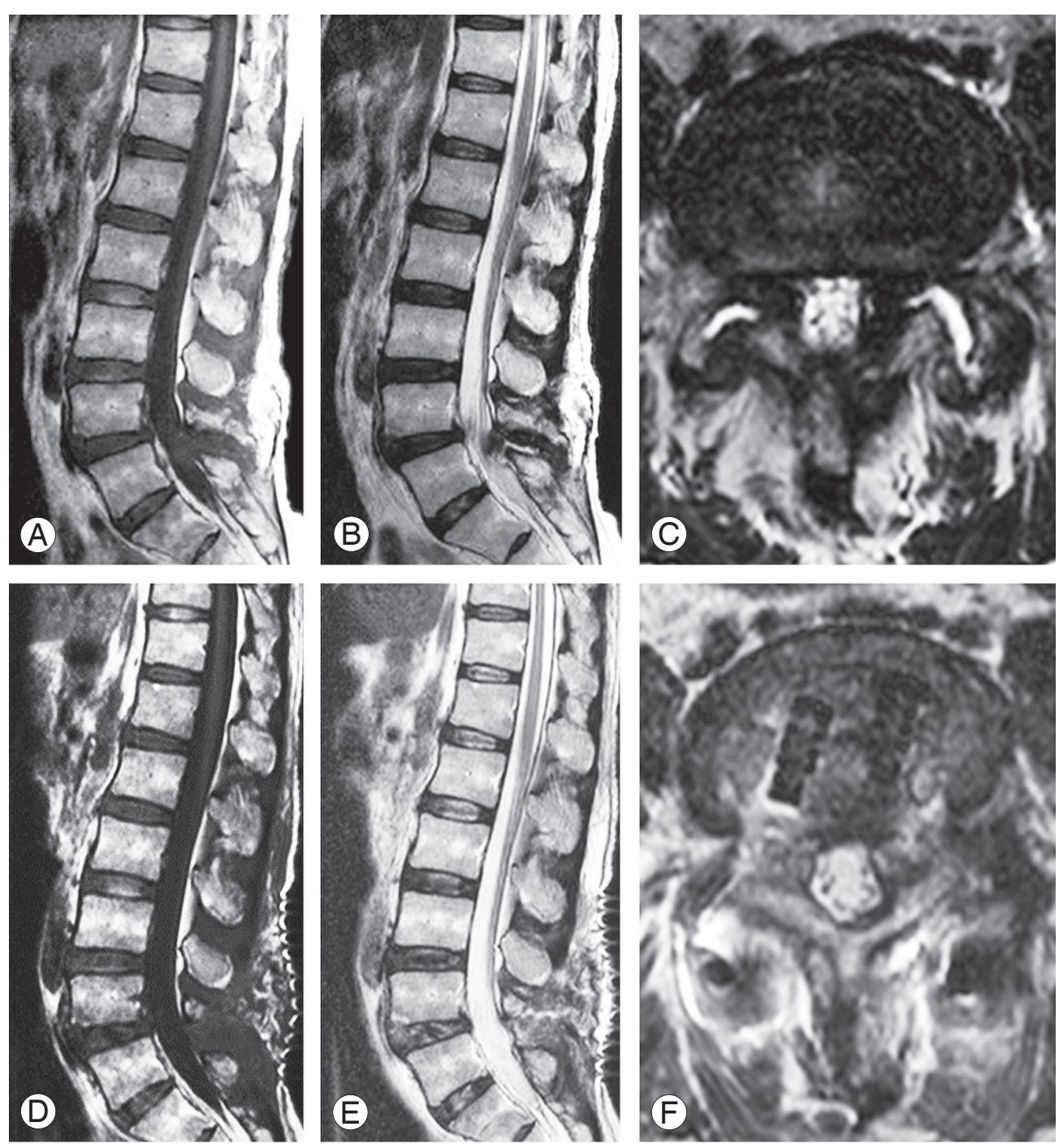

Fig. 2. An illustrative case of "grade 0" spinal subdural lesion (SSDL) according to our grading system. There was no evidence of postoperative changes in the dura mater on T2-weighted sagittal or axial magnetic resonance (MR) images. (A) preoperative T1-weighted sagittal MR image, (B) preoperative T2-weighted sagittal MR image, (C) preoperative T2-weighted axial MR image, (D) postoperative T1-weighted sagittal MR image, (E) postoperative T2-weighted sagittal MR image, and (F) postoperative T2-weighted axial MR image.

of SOL, a grade 1 SSDL was considered to be an iatrogenic arachnoid cyst and a grade 2 SSDL was considered to be SSDH. The level and number of affected segments were recorded for both grade 1 and grade 2 SSDLs. The clinical course of SSDLs was recorded for patients who underwent follow-up MRI for reasons other than SSDL. Intra- and inter-observer reliability of the grading system was evaluated for 30 randomly selected MR images by two spinal surgeons who were blinded to patients' clinical information (Y.N. [R1] and S.T. [R2]). For intra-rater reliability, grading was repeated at an interval of 2 weeks. Reproducibility of the grading system was evaluated using the kappa coefficient, with both intra- and inter-rater reliability being acceptable: intra-rater kappa of 0.91 for R1 and 0.81 for R2 and inter-rater kappa of 0.83 .

\section{Statistical analysis}

Differences between patients with and without SSDLs were evaluated using univariate analysis, with the Fisher's exact test for categorical variables and the Mann-Whitney $U$ test for continuous variables. Risk factors for SSDH were identified using logistic regression analysis. The following factors were included in the multivariate analysis 

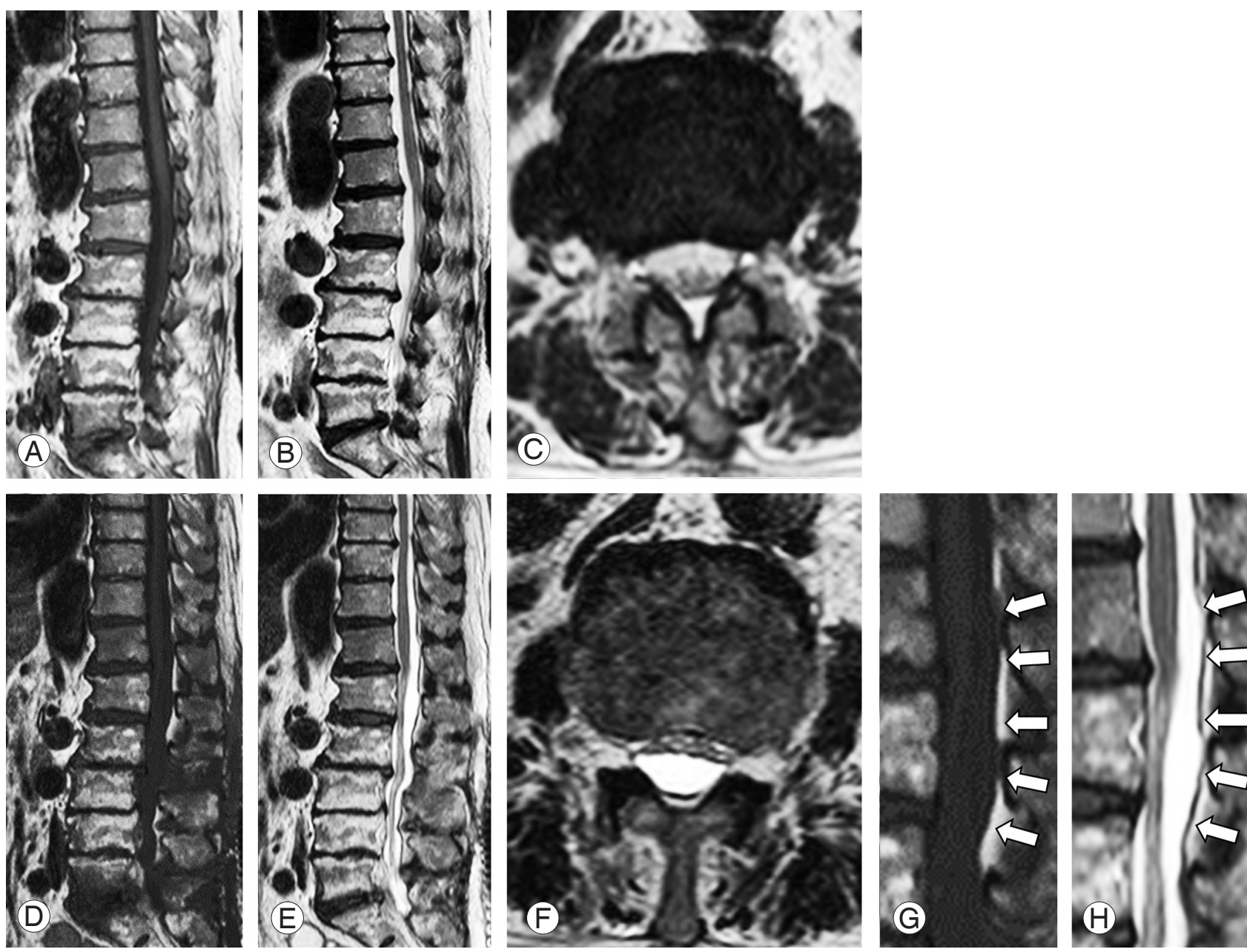

Fig. 3. An illustrative case of "grade 1" spinal subdural lesion (SSDL) according to our grading system. Space-occupying lesion (SOL) was identified within the dura mater. The signal intensity of SOL was equivalent to that of cerebrospinal fluid (T1 hypo-intensity/T2 hyper-intensity). These SOLS are considered arachnoid cysts. (A). preoperative T1-weighted sagittal magnetic resonance (MR) image, (B) preoperative T2-weighted sagittal MR image, (C) preoperative T2-weighted axial MR image, (D) postoperative T1-weighted sagittal MR image, (E) postoperative T2-weighted sagittal MR image, (F) postoperative T2-weighted axial MR image, (G) magnified postoperative T1-weighted sagittal MR image, and (H) magnified postoperative T2-weighted sagittal MR image. Arrows indicate fluid collection within the dura mater.

as confounders: age, sex, body mass index (weight $[\mathrm{kg}] /$ height ${ }^{2}\left[\mathrm{~m}^{2}\right]$ ), primary disease (degenerative spondylosis, spinal canal stenosis, lumbar disk herniation, spondylolytic spondylolisthesis, adjacent segment disease, and other diseases), use of anticoagulants, type of surgical procedure (PLIF, BPL, UPL, UPLWD, and endoscopic surgery), previous surgery at the same level, incidental durotomy, length of surgery, and estimated blood loss.

A logistic regression analysis was also performed to identify risk factors for postoperative SSDLs. Parameter significance was evaluated using univariate analysis. Factors with a $p$-value of $<0.05$ in the univariate analysis were included in the multivariate analysis. To investigate the influence of SSDLs on postoperative pain, we addition- ally compared the dosage of diclofenac suppository use between patients with and without SSDLs using the nonparametric Mann-Whitney $U$ test. All statistical analyses were conducted using SPSS ver. 21 (SPSS, Chicago, IL, USA).

\section{Results}

\section{Prevalence of postoperative SSDLs on MRI}

Postoperative SSDLs were identified in 123 patients (30.0\%), with $50(12.2 \%)$ and $73(17.8 \%)$ patients being classified with grade 1 and 2 SSDLs, respectively. All grade 2 SSDLs were characterized by T1-weighted iso- 

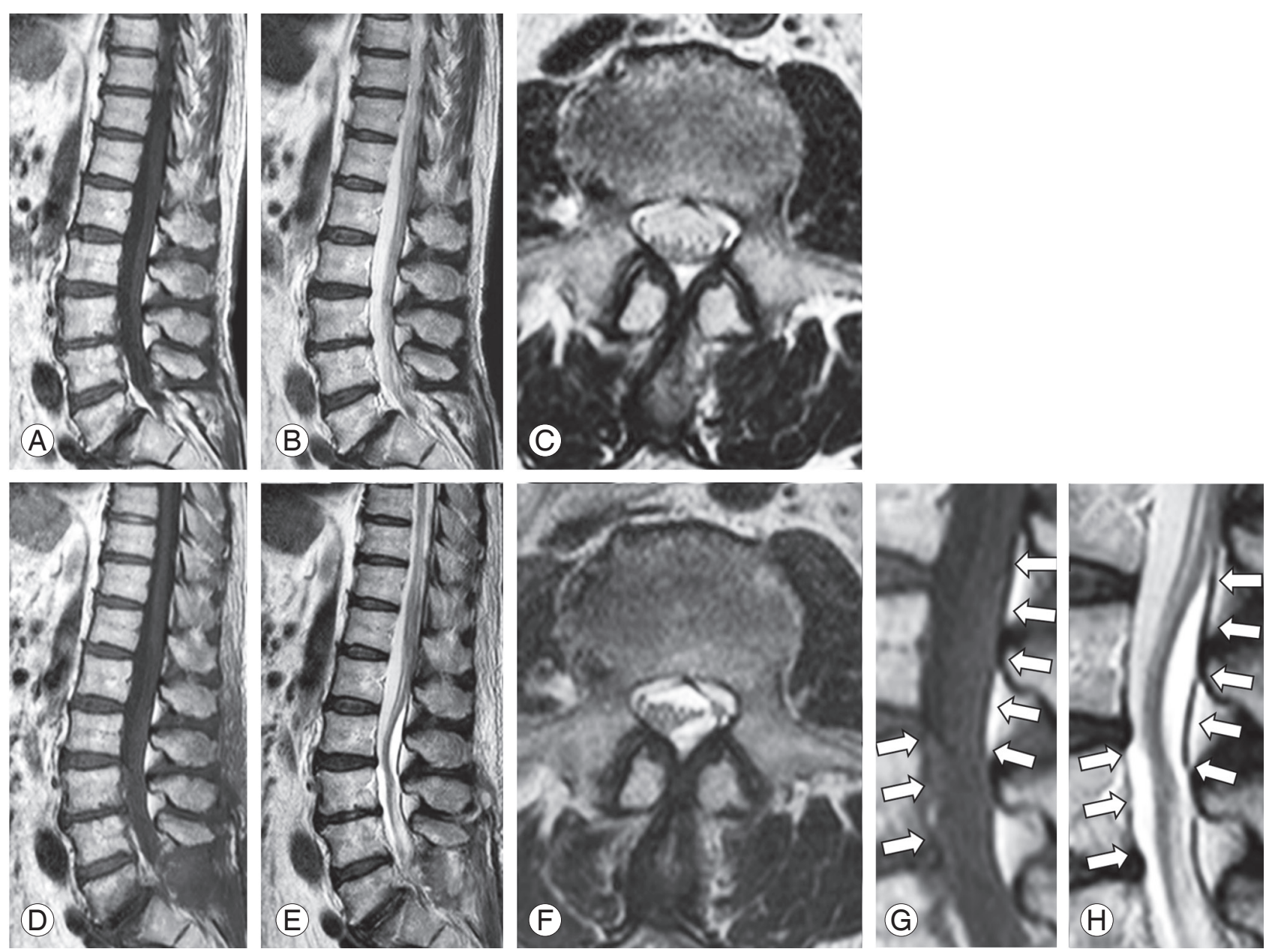

Fig. 4. An illustrative case of "grade 2" spinal subdural lesion (SSDL) according to our grading system. Space-occupying lesion (SOL) was identified within the dura mater. The signal intensity of SOL was different from that of cerebrospinal fluid. These SOLs are more likely to be spinal subdural hematomas than arachnoid cysts. (A) Preoperative T1-weighted sagittal magnetic resonance (MR) image, (B) preoperative T2-weighted sagittal MR image, (C) preoperative T2-weighted axial MR image, (D) postoperative T1-weighted sagittal MR image, (E) postoperative T2-weighted sagittal MR image, (F) postoperative T2-weighted axial MR image, (G) magnified postoperative T1-weighted sagittal MR image, and (H) magnified postoperative T2-weighted sagittal MR image. Arrows indicate fluid collection within the dura mater.

Table 1. Level and number of affected segments by postoperative SSDH

\begin{tabular}{lcccccccccc} 
Grade & T9/10 & T10/11 & T11/12 & T12/L1 & $L 1 / 2$ & $L 2 / 3$ & $L 3 / 4$ & $L 4 / 5$ & $L 5 / S$ & $\begin{array}{c}\text { Affected } \\
\text { segments } \\
\text { (no. of patients) }\end{array}$ \\
\hline Grade $1(n=50)$ & $1(2.0)$ & $2(4.0)$ & $3(6.0)$ & $10(20.0)$ & $24(48.0)$ & $22(44.0)$ & $12(24.0)$ & $20(40.0)$ & $24(48.0)$ & $2.4 \pm 1.5$ \\
Grade 2 $(n=73)$ & $1(1.4)$ & $2(2.7)$ & $4(5.5)$ & $11(15.1)$ & $32(43.8)$ & $37(50.7)$ & $33(45.2)$ & $35(47.9)$ & $24(32.9)$ & $2.5 \pm 1.5$ \\
\hline
\end{tabular}

Values are presented as number of patients (\%, prevalence rate of postoperative SSDH at each level).

SSDH, spinal subdural hematoma.

to hyper-intensity and T2-weighted hyper-intensity and were, therefore, distinct from CSF. Among the 123 cases of SSDLs identified, only one was symptomatic, requiring hematoma evacuation because of the development of incomplete paraplegia. The level and number of segments affected by SSDLs are reported in Table 1. Grade 1 lesions affected $2.4 \pm 1.5$ segments (range, 1-6 segments) and grade 2 lesions affected $2.5 \pm 1.5$ segments (range, $1-6$ segments). The prevalence of postoperative SSDLs according to type of surgical procedure is reported in Table 2. The 
prevalence rates of SSDLs after PLIF and BPL were 29.5\% and $39.8 \%$, respectively, and no incidence of SSDLs was noted after UPL and UPLWD. There was no difference in the dosages of diclofenac suppository between patients with $(77 \pm 97 \mathrm{mg})$ and without $(75 \pm 109 \mathrm{mg} ; p=0.495)$ SSDLs. Follow-up MRI was performed in 55 patients at 8.6 \pm 7.6 months (range, $0-33$ months) postoperatively,

Table 2. Surgical details and the prevalence of SSDL by type of procedure

$\begin{array}{lccc}\text { Surgical } & \text { SSDL } & \text { No-SSDL } & \text { Prevalence } \\ \text { procedure } & (n=123) & (n=287) & (\%)\end{array}$

PLIF

\begin{tabular}{|c|c|c|c|}
\hline 2PLIF & 12 & 14 & 46.1 \\
\hline 2PLIF+1BPL & 1 & 2 & 33.3 \\
\hline 1PLIF & 30 & 108 & 21.7 \\
\hline 1PLIF+1UPL & 1 & 3 & 25.0 \\
\hline 1PLIF+1BPL & 15 & 28 & 34.9 \\
\hline 1PLIF+2BPL & 9 & 11 & 45.0 \\
\hline 1PLIF+3BPL & 2 & 1 & 66.6 \\
\hline Total & 70 & 167 & 29.5 \\
\hline \multicolumn{4}{|l|}{ BPL } \\
\hline $1 \mathrm{BPL}$ & 19 & 44 & 30.2 \\
\hline $1 \mathrm{BPL}+1 \mathrm{UPL}$ & 1 & 3 & 25.0 \\
\hline $2 \mathrm{BPL}$ & 22 & 18 & 55.0 \\
\hline $2 B P L+1 U P L$ & 0 & 1 & 0.0 \\
\hline 3BPL & 4 & 5 & 44.4 \\
\hline 3BPL+1UPL & 0 & 1 & 0.0 \\
\hline $4 \mathrm{BPL}$ & 1 & 3 & 25.0 \\
\hline $5 B P L$ & 1 & 0 & 100.0 \\
\hline 1End-BPL & 5 & 5 & 50.0 \\
\hline Total & 53 & 80 & 39.8 \\
\hline \multicolumn{4}{|l|}{ UPL } \\
\hline $1 \mathrm{UPL}$ & 0 & 5 & 0.0 \\
\hline $2 U P L$ & 0 & 1 & 0.0 \\
\hline 1End-UPL & 0 & 1 & 0.0 \\
\hline Total & 0 & 7 & 0.0 \\
\hline \multicolumn{4}{|l|}{ UPLWD } \\
\hline 1UPLWD & 0 & 29 & 0.0 \\
\hline 1End-UPLWD & 0 & 4 & 0.0 \\
\hline Total & 0 & 33 & 0.0 \\
\hline
\end{tabular}

SSDH, spinal subdural hematoma; PLIF, posterior lumbar interbody fusion; BPL, bilateral partial laminectomy; UPL, unilateral partial laminectomy; End-, endoscopic procedure; UPLWD, unilateral partial laminectomy with discectomy. with evidence of SSDL regression noted in all cases.

\section{Risk factor analysis for developing postoperative SSDLs}

Univariate analysis identified the presence of BPL and UPL and length of surgery as significant risk factors for SSDLs (Table 3). The following covariates (univariate, $p<0.05)$ were included in the multivariate analysis: age, primary disease, type of procedure (BPL, UPL, and discectomy), and length of surgery. Results of the multivariate logistic regression analysis are reported in Table 4. BPL was identified as an independent risk factor for SSDLs (odds ratio, 1.52; 95\% confidence interval [CI], 1.20-1.92; $p<0.001$ ), whereas UPL was identified as a protective factor that lowered the risk for SSDLs (odds ratio, $0.11 ; 95 \%$ CI, 0.03-0.46; $p=0.002$ ).

\section{Symptomatic case of SSDL requiring hematoma evacuation}

Among our 123 cases of SSDLs, only one was clinically symptomatic. This was the case of an 87-year-old male patient who was admitted to our institution with a diagnosis of osteoporotic vertebral collapse of L2. He had a history of coronary artery disease, which had been treated with low-dose aspirin. Blood tests were negative for coagulation abnormalities. Aspirin was discontinued at 7 days prior to surgery. The patient underwent PLIF at the L2-L3 level. Surgery proceeded uneventfully, except for a slightly higher than expected volume of blood loss $(600 \mathrm{~mL})$. On postoperative day 4 , the patient developed severe weakness on left hip flexion and knee extension, followed by a progressive bilateral neurological deterioration below L3. The patient could no longer raise his legs or flex his knees. MRI was performed on an urgent basis, showing evidence of severe compression of the cauda equina by a large T1 hypo- and T2 hyper-intense collection within the dura mater, extending from approximately T10 to S1 (Fig. 5). To determine the precise location of SOL within the dura mater, computed tomography (CT) was performed via lumbar puncture. Xanthochromic CSF was identified on lumbar puncture, with subsequent CT myelography confirming the presence of a lesion situated on the posterior wall within the dura mater. Based on this evidence, SOL was diagnosed as an iatrogenic intradural spinal arachnoid cyst occurring because of an unknown durotomy incident [15]. An emergency revision surgery was per- 
Table 3. Comparison of patients with and without postoperative SSDL

\begin{tabular}{|c|c|c|c|}
\hline Variable & SSDL (n=123) & No-SSDH $(n=287)$ & $p$-value \\
\hline Age & $72.6 \pm 9.5$ & $69.4 \pm 11.7$ & $0.012^{*}$ \\
\hline Sex & M: 69, F: 54 & M: 135, F: 152 & 0.106 \\
\hline Height (cm) & $157.8 \pm 10.0$ & $157.3 \pm 9.7$ & 0.603 \\
\hline Weight (kg) & $60.3 \pm 12.1$ & $60.0 \pm 11.7$ & 0.635 \\
\hline Body mass index $\left(\mathrm{kg} / \mathrm{m}^{2}\right)$ & $24.1 \pm 3.7$ & $24.1 \pm 3.6$ & 0.892 \\
\hline Anticoagulant therapy & $38(30.9)$ & $68(23.7)$ & 0.140 \\
\hline Primary disease & & & $0.002^{*}$ \\
\hline Degenerative spondylolisthesis & 45 & 113 & \\
\hline Spinal canal stenosis & 61 & 97 & \\
\hline Lumbar disc herniation & 4 & 39 & \\
\hline Spondylolytic spondylolisthesis & 6 & 15 & \\
\hline Adjacent segment disease & 3 & 10 & \\
\hline Other diseases & 4 & 13 & \\
\hline \multicolumn{4}{|l|}{ Surgical procedure } \\
\hline \multicolumn{4}{|l|}{ No. of levels } \\
\hline PLIF & $0.7 \pm 0.7$ & $0.6 \pm 0.6$ & 0.759 \\
\hline BPL & $1.1 \pm 1.0$ & $0.6 \pm 0.8$ & $<0.001^{*}$ \\
\hline UPL & $0.0 \pm 0.1$ & $0.2 \pm 0.4$ & $<0.001^{*}$ \\
\hline Discectomy & $7(5.7)$ & $46(16.0)$ & $0.004^{*}$ \\
\hline Endoscopic surgery & $5(4.1)$ & $10(3.5)$ & 0.778 \\
\hline Reoperation at the same level & $4(3.3)$ & $27(9.4)$ & $0.040^{*}$ \\
\hline Incidental durotomy & $12(9.8)$ & $29(10.1)$ & $>0.99$ \\
\hline Length of surgery & $1.7 \pm 0.8$ & $1.4 \pm 0.7$ & $<0.001^{*}$ \\
\hline Estimated blood loss (mL) & $130 \pm 161$ & $125 \pm 195$ & 0.701 \\
\hline Operative time (min) & $117 \pm 56$ & $107 \pm 53$ & 0.118 \\
\hline
\end{tabular}

Values are presented as mean \pm standard deviation (SD) or number (\%).

Fisher exact test or Mann-Whitney $U$ test was used to compare each factor between patients with and without postoperative SSDL.

SSDL, spinal subdural lesion; M, male; F, female; PLIF, posterior lumbar interbody fusion; BPL, bilateral partial laminectomy; UPL, unilateral partial laminectomy.

${ }^{*} p<0.05$.

Table 4. Logistic regression analysis of risk factors of postoperative SSDL

\begin{tabular}{lcc} 
Variable & $\begin{array}{c}\text { Odds ratio } \\
\text { (95\% confidence interval })\end{array}$ & $p$-value \\
BPL & $1.52(1.20-1.92)$ & $<0.001^{*}$ \\
UPL & $0.11(0.03-0.46)$ & $0.002^{*}$ \\
\hline
\end{tabular}

The multivariate model included variables with a $p$-value $<0.05$ on univariate analysis. The multivariate model was adjusted for age, BPL, UPL, discectomy reoperation at the same level, and length of surgical level.

SSDL, spinal subdural lesion; BPL, bilateral partial laminectomy; UPL, unilateral partial laminectomy.

${ }^{*} p<0.05$. formed. Intraoperatively, no trace of incidental durotomy was identified, and there was no significant epidural hematoma. The dura, however, was tense and discolored. After opening the dural sac at the L2-L3 level, a clot was observed in the subdural space (Fig. 6A). The clot was evacuated en bloc using forceps (Fig. 6B). After evacuation, an intact arachnoid membrane was noted (Fig. 6C), with clear CSF having been observed after fenestration of the arachnoid membrane (Fig. 6D). Follow-up MRI was performed on postoperative day 2 , showing a decrease in the volume of the posterior compartment (Fig. 7). Three months after revision surgery, the patient was walking without assistance despite the persistence of slight weak- 

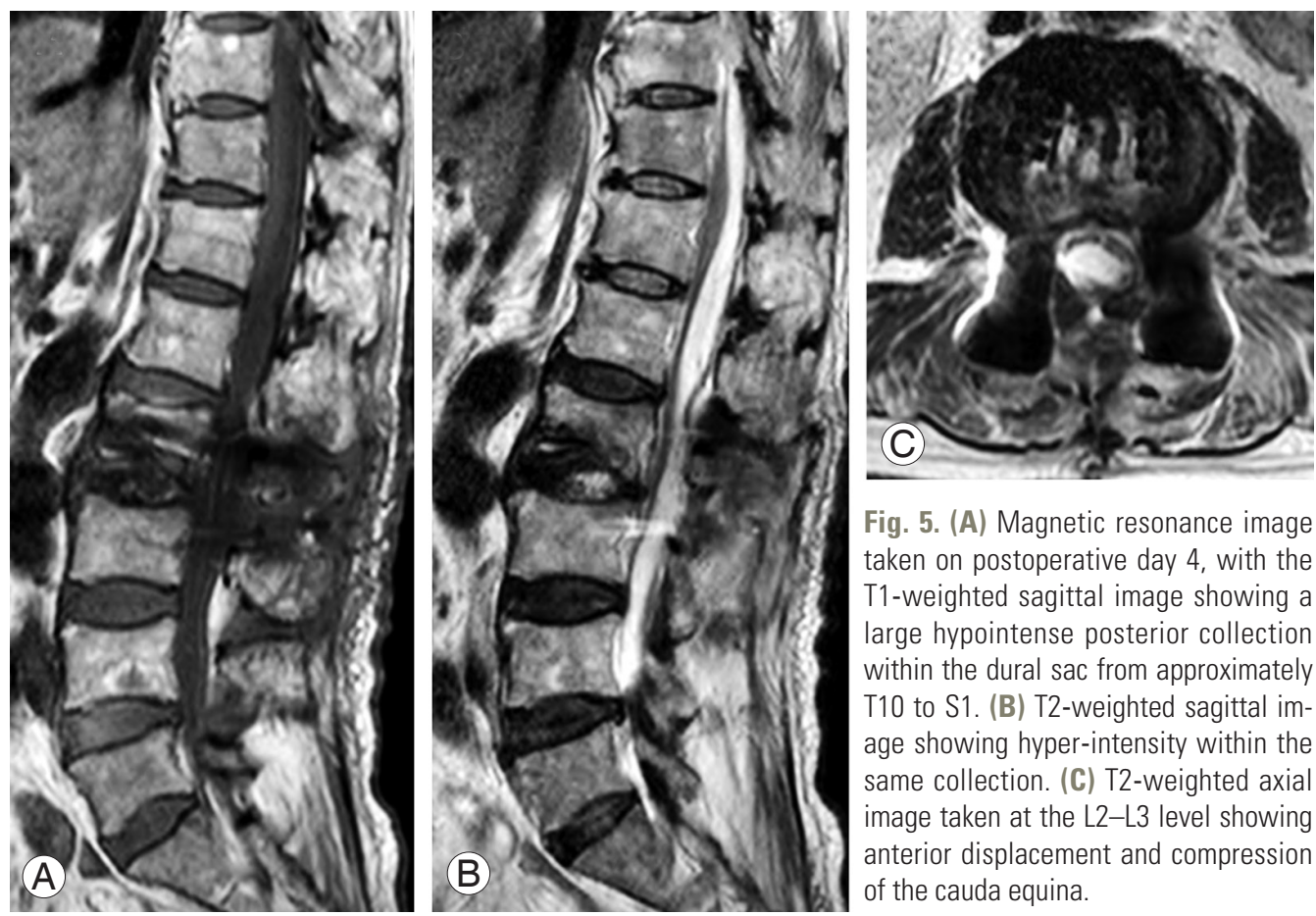

Fig. 5. (A) Magnetic resonance image taken on postoperative day 4, with the T1-weighted sagittal image showing a large hypointense posterior collection within the dural sac from approximately T10 to S1. (B) T2-weighted sagittal image showing hyper-intensity within the same collection. (C) T2-weighted axial image taken at the $\mathrm{L} 2-\mathrm{L} 3$ level showing anterior displacement and compression of the cauda equina.
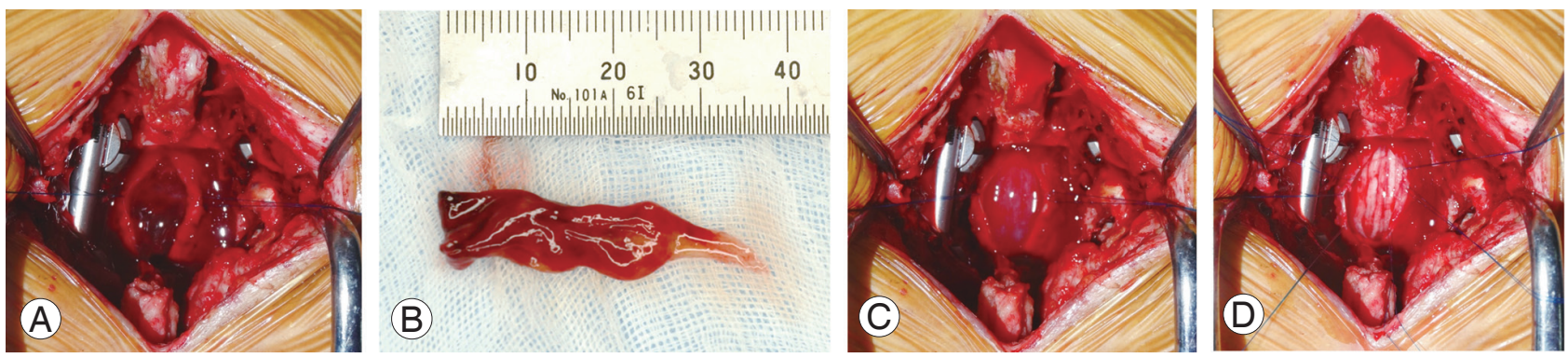

Fig. 6. (A) Intraoperative photograph showing an extensive mass of clotted blood that was identified when the dura mater was opened. (B, C) Following en bloc evacuation of the clotted blood, the distended normal arachnoid sac was exposed. (D) Fenestration of the arachnoid membrane was performed.

ness in left hip flexion and knee extension.

\section{Discussion}

Although spinal epidural hematoma is a relatively common cause of symptomatic neural compression during the early postoperative period following spinal surgery, hematoma evacuation is required in $<0.5 \%$ of cases $[1,2]$. In contrast, SSDLs, including the arachnoid cyst and subdural hematoma, that develop following spinal surgery are seldom symptomatic and require reoperation. Therefore, there are few reports regarding these pathologies $[5,10,11,15,16]$.

SSDH was first identified by Schiller et al. [17] as a complication of hemophilia. A large review of all spinal hematomas reported that SSDH is rare, with its incidence rate of $4.1 \%$ ( 25 of 613 cases) being substantively lower than the rates for spinal epidural hematoma (75\%; 461 of 613 cases) and subarachnoid hematoma (15.7\%; 96 of 613 cases) [7]. The paucity of vessels within the subdural space explains the infrequent occurrence of SSDH [9]. Considering the paucity of blood vessels that traverse the spinal subdural space, it has been suggested that SSDH originates in the vascular subarachnoid space, with a subsequent subdural dissection [18-20]. The blood from the ruptured vessels in the subdural space would be cleared by CSF, leaving behind an isolated SSDH [18-20]. This proposed pathway for the development of SSDH is supported 

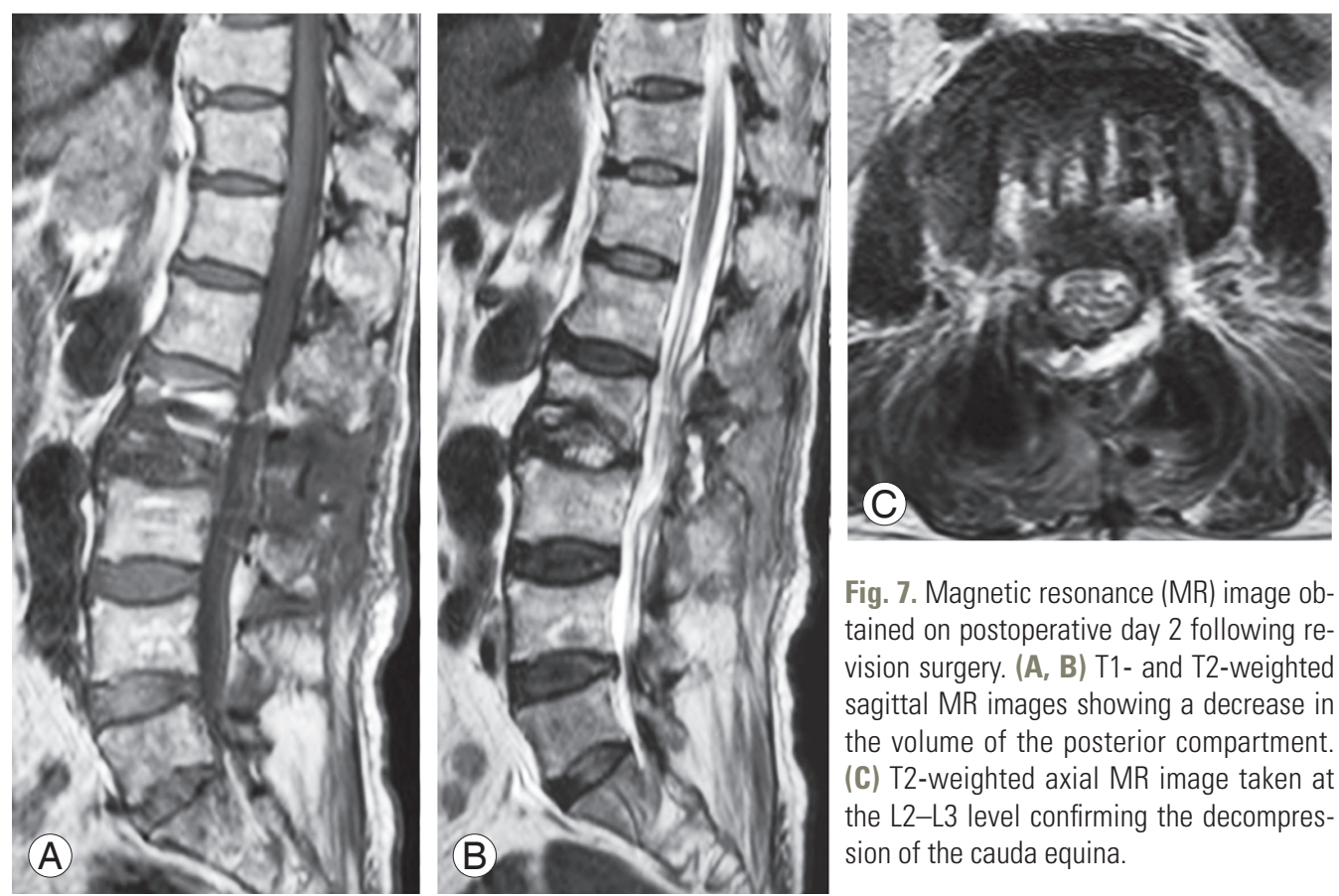

Fig. 7. Magnetic resonance (MR) image obtained on postoperative day 2 following revision surgery. (A, B) T1- and T2-weighted sagittal MR images showing a decrease in the volume of the posterior compartment. (C) T2-weighted axial MR image taken at the $\mathrm{L} 2-\mathrm{L} 3$ level confirming the decompression of the cauda equina.

by our findings of xanthochromic CSF noted during a lumbar puncture that was performed for myelography. We observed clear CSF after fenestration of the arachnoid membrane at the time of reoperation in our case.

SSDH most frequently occurs in patients with coagulopathy after lumbar puncture $[20,21]$ or with trauma $[19,22,23]$ and rarely occurs spontaneously in patients receiving anticoagulants or in those who have hematological disorders, vascular malformations, or tumors $[3,4,7,9]$. SSDH following spinal surgery is an extremely rare complication, with only five cases having been reported in the literature $[5,10,11,16]$. In all cases, lesions became symptomatic within 10 (range, 0-10; mean, 3.8) days of surgery. Surgery-related trauma, in the form of incidental durotomy or traumatic blunt dissection, may be the cause of SSDH after lumbar surgery [5,10]. Gehri et al. [5] attributed the occurrence of SSDH to a minor dural tear during surgery which was repaired intraoperatively. Chang et al. [10] reported on a case in which the dura was adherent to the posterior spinal element and required blunt dissection during laminectomy, leading to trauma of the delicate dural tissue. Incidental durotomy or previous operation at the same level as the current surgery was not identified as a risk factor for SSDL or SSDH in our retrospective analysis. In the present clinical case, the patient had no predisposing factors, and the dura was not adherent.

The development of a spinal arachnoid cyst after spinal surgery is also extremely rare, with only two cases having been reported [15]. Nottmeier et al. [15] reported two cases of symptomatic arachnoid cyst following lumbar spine surgery, which required cyst fenestration. In both cases, the lesions became symptomatic at $>2$ weeks postoperatively, with symptoms typically developing more slowly than those for SSDH. In both of these cases, the cyst was deemed to have formed after injury to the arachnoid membrane during incidental durotomy. In comparison, in our case series, $12 \%$ patients had the same MRI findings as those for spinal arachnoid cyst, although no patients developed symptoms that required cyst fenestration.

A definite differentiation of hematomas from cysts within CSF is difficult based on MRI assessment alone $[7,24]$. In our cases series, we considered most grade 1 and 2 SSDLs to be iatrogenic arachnoid cysts, with some grade 2 SSDLs being SSDHs. The pathway of SSDL formation after spinal surgery is probably initiated by an injury to the arachnoid membrane during intraoperative manipulation of neural tissue. This injury allows CSF to collect in the subdural space between the dura mater and arachnoid membrane. If vessels beneath the arachnoid membrane are also injured, blood will accumulate in the subdural space, resulting in the development of SSDH. Owing to the sufficiency of the subdural space for the cauda equina at the lumbar spine, these SSDLs are rarely symptomatic. In our case series, a postoperative SSDL was identified in 
$30 \%$ of our 410 cases, with only one of these being symptomatic and requiring reoperation.

Although we had hypothesized a priori that PLIF would probably increase the risk for SSDLs, BPL was identified as an independent risk factor for SSDLs on multivariate analysis. Because bilateral superior articular facets are generally preserved in BPL, semi-circumferential decompression must be performed within a more confined spinal canal. Under this confined condition, injury to the arachnoid tissue may occur during manipulation of the dura mater tissue, with the dura mater being deformed against the nerve retractor and contralateral superior articular facet, particularly during resection of the inner edge of the superior articular facet or residual ligamentum flavum. Conversely, during PLIF procedure, dural retraction may be accomplished with less strain than anticipated even if large dural displacement is required because total facetectomy provides more working space and a wideangle approach to the neural structures [12]. In a large cohort study, the incidence of dural tears was higher when lumbar spine decompression was performed alone than when it was performed with instrumented fusion $(8.5 \%$ versus 5.5\%) [25]. The absence of SSDL formation in UPL cases reflects the inability to perform substantive dural retraction because of restricted access to the dura within the relatively small fenestration window in the inter-laminar space. Therefore, although UPL requires additional discectomy compared with BPL and PLIF, the strain applied to the dura during this procedure will remain lower than that applied to the dura during BPL and PLIF. Given these observations, it is important to take extreme care during manipulation of the dura to avoid excessive dural traction and the possible development of SSDL. These findings support the results of our risk factor analysis.

The limitations of our study need to be acknowledged. Foremost, this is a retrospective study with patients identified from a single institution. SSDL was diagnosed using routine preoperative and postoperative MR images, which included only sagittal T1- and T2-weighted sequences and axial T2-weighted sequences. Our diagnostic accuracy could be improved by including axial T1-weighted or other sequences, such as the $\mathrm{T} 2{ }^{\star}$-weighted gradient echo or fluid attenuated inversion recovery [26]. Specific information regarding postoperative pain was not consistently available in medical records and was, therefore, not included in our analysis. Rather, we used the dosage of diclofenac suppository administered over the first 7 post- operative days as a surrogate measure of pain. We believe that SSDL, particularly SSDH, may be associated with postoperative pain, particularly in the lower extremities. Although we did not identify a significant difference in diclofenac dosage between patients with and without SSDL, additional prospective studies are required to clarify this relationship.

\section{Conclusions}

Although SSDLs following lumbar spine surgery have been considered to be extremely rare, we identified SSDLs in $30 \%$ of our 410 cases of lumbar spine surgery. Among these 123 cases of SSDL, 41\% (50 patients) were considered to be iatrogenic arachnoid cysts and 59\% (73 patients) were considered as possible SSDHs. Therefore, we are the first to demonstrate that the prevalence of SSDH might be higher than that expected previously. However, the association of clinical symptoms with SSDLs is rare, with only 1 of our 123 cases requiring reoperation because of a declining neurological status. BPL increased the risk for SSDL development, whereas UPL was identified as a protective factor. SSDL development is closely related to the strain applied to the dura mater during intraoperative manipulation. Therefore, extreme care is required to avoid excessive dural traction during surgery to lower the risk for SSDLs.

\section{Conflict of Interest}

No potential conflict of interest relevant to this article was reported.

\section{Acknowledgments}

The authors give special thanks to Yukiko Miyake (Medical secretary, Department of Orthopaedic Surgery, National Hospital Organization Osaka Medical Center) for assisting with the collection of patient data.

\section{ORCID}

Yukitaka Nagamoto: https://orcid.org/0000-0002-7993-3959

Shota Takenaka: https://orcid.org/0000-0001-9956-5914

Hiroyuki Aono: https://orcid.org/0000-0002-3520-3292 


\section{References}

1. Aono H, Ohwada T, Hosono N, et al. Incidence of postoperative symptomatic epidural hematoma in spinal decompression surgery. J Neurosurg Spine 2011;15:202-5.

2. Yamada K, Abe Y, Satoh S, Yanagibashi Y, Hyakumachi T, Masuda T. Large increase in blood pressure after extubation and high body mass index elevate the risk of spinal epidural hematoma after spinal surgery. Spine (Phila Pa 1976) 2015;40:1046-52.

3. Domenicucci M, Ramieri A, Ciappetta P, Delfini R. Nontraumatic acute spinal subdural hematoma: report of five cases and review of the literature. J Neurosurg 1999;91(1 Suppl):65-73.

4. Edelson RN, Chernik NL, Posner JB. Spinal subdural hematomas complicating lumbar puncture. Arch Neurol 1974;31:134-7.

5. Gehri R, Zanetti M, Boos N. Subacute subdural haematoma complicating lumbar microdiscectomy. J Bone Joint Surg Br 2000;82:1042-5.

6. Jonsson LO, Einarsson P, Olsson GL. Subdural haematoma and spinal anaesthesia: a case report and an incidence study. Anaesthesia 1983;38:144-6.

7. Kreppel D, Antoniadis G, Seeling W. Spinal hematoma: a literature survey with meta-analysis of 613 patients. Neurosurg Rev 2003;26:1-49.

8. Reinsel TE, Goldberg E, Granato DB, Wilkinson S, Penn R. Spinal subdural hematoma: a rare cause of recurrent postoperative radiculopathy. J Spinal Disord 1993;6:62-7.

9. Russell NA, Benoit BG. Spinal subdural hematoma: a review. Surg Neurol 1983;20:133-7.

10. Chang KC, Samartzis D, Luk KD, Cheung KM, Wong YW. Acute spinal subdural hematoma complicating lumbar decompressive surgery. Evid Based Spine Care J 2012;3:57-62.

11. Lykissas MG, Aichmair A, Herzog RJ, Schroeder JE, Huang RC, Farmer J. Spinal subdural hematoma following lumbar decompressive surgery: a report of two cases. Wien Klin Wochenschr 2015;127:71-4.

12. Okuda S, Oda T, Miyauchi A, Haku T, Yamamoto T, Iwasaki M. Surgical outcomes of posterior lumbar interbody fusion in elderly patients: surgical technique. J Bone Joint Surg Am 2007;89 Suppl 2 Pt.2:310-20.

13. Takenaka S, Tateishi K, Hosono N, Mukai Y, Fuji T. Preoperative retrolisthesis as a risk factor of postde- compression lumbar disc herniation. J Neurosurg Spine 2016;24:592-601.

14. Minamide A, Yoshida M, Yamada H, et al. Endoscope-assisted spinal decompression surgery for lumbar spinal stenosis. J Neurosurg Spine 2013;19:66471.

15. Nottmeier EW, Wharen RE, Patel NP. Iatrogenic intradural spinal arachnoid cyst as a complication of lumbar spine surgery. J Neurosurg Spine 2009;11:344-6.

16. Chung T, Thien C, Wang YY. A rare cause of postoperative paraplegia in minimally invasive spine surgery. Spine (Phila Pa 1976) 2014;39:E228-30.

17. Schiller F, Neligan G, Budtz-olsen O. Surgery in haemophilia; a case of spinal subdural haematoma producing paraplegia. Lancet 1948;2:842-5.

18. Masdeu JC, Breuer AC, Schoene WC. Spinal subarachnoid hematomas: clue to a source of bleeding in traumatic lumbar puncture. Neurology 1979;29:872-6.

19. Swann KW, Ropper AH, New PF, Poletti CE. Spontaneous spinal subarachnoid hemorrhage and subdural hematoma. Report of two cases. J Neurosurg 1984;61:975-80.

20. Vinters HV, Barnett HJ, Kaufmann JC. Subdural hematoma of the spinal cord and widespread subarachnoid hemorrhage complicating anticoagulant therapy. Stroke 1980;11:459-64.

21. Guthikonda M, Schmidek HH, Wallman LJ, Snyder TM. Spinal subdural hematoma: case report and review of the literature. Neurosurgery 1979;5:614-6.

22. Stewart DH Jr, Watkins ES. Spinal cord compression by chronic subdural hematoma: case report. J Neurosurg 1969;31:80-2.

23. Zilkha A, Nicoletti JM. Acute spinal subdural hematoma: case report. J Neurosurg 1974;41:627-30.

24. Chakeres DW, Bryan RN. Acute subarachnoid hemorrhage: in vitro comparison of magnetic resonance and computed tomography. AJNR Am J Neuroradiol 1986; 7:223-8.

25. Stromqvist F, Jonsson B, Stromqvist B; Swedish Society of Spinal Surgeons. Dural lesions in decompression for lumbar spinal stenosis: incidence, risk factors and effect on outcome. Eur Spine J 2012;21:825-8.

26. Kuker W, Thiex R, Friese S, et al. Spinal subdural and epidural haematomas: diagnostic and therapeutic aspects in acute and subacute cases. Acta Neurochir (Wien) 2000;142:777-85. 